\title{
Optogenetic suppression of the medial septum impairs working memory maintenance
}

\author{
Zachary M. Gemzik, Margaret M. Donahue, and Amy L. Griffin \\ Department of Psychological and Brain Sciences, University of Delaware, Newark, Delaware 19711, USA
}

\begin{abstract}
Spatial working memory (SWM) is the ability to encode, maintain, and retrieve spatial information over a temporal gap, and relies on a network of structures including the medial septum (MS), which provides critical input to the hippocampus. Although the role of the MS in SWM is well-established, up until recently, we have been unable to use temporally precise circuit manipulation techniques to examine the specific role of the MS in SWM, particularly to distinguish between encoding, maintenance, and retrieval. Here, we test the hypothesis that the MS supports the maintenance of spatial information over a temporal gap using precisely timed optogenetic suppression delivered during specific portions of three different tasks, two of which rely on SWM and one that does not. In experiment 1, we found that MS optogenetic suppression impaired choice accuracy of a SWM dependent conditional discrimination task. Moreover, this deficit was only observed when MS suppression was delivered during the cue-sampling, but not the cue-retrieval, portion of the trial. There was also no deficit when MS neurons were optogenetically suppressed as rats performed a SWM-independent variant of the task. In experiment 2, we tested whether MS suppression affected choice accuracy on a delayed nonmatch to position (DNMP) task when suppression was limited to the sample, delay, and choice phases of the task. We found that MS suppression delivery during the delay phase of the DNMP task, but not during the sample or choice phases, impaired choice accuracy. Our results collectively suggest that the MS plays an important role in SWM by maintaining task-relevant information over a temporal delay.
\end{abstract}

Successful spatial working memory (SWM) requires trial-specific information to be first encoded, maintained over a short delay, and later retrieved at the precise time that it is needed. This critical cognitive function is supported in part by the medial septum (MS), a subcortical region that is reciprocally connected to the hippocampal formation (Frotscher and Leranth 1985; Freund and Antal 1988; Toth et al. 1993; Hajszan et al. 2004; Manseau et al. 2005; Sun et al. 2014; Fuhrmann et al. 2015; Leao et al. 2015; Muller and Remy 2018). Disruption of MS input to the hippocampus through MS or fimbria/fornix lesions or reversible inactivation impairs spatial memory tasks (Sutherland and Rodriguez 1989; Mizumori et al. 1990; Poucet and Buhot 1994; Whishaw and Jarrard 1995). Moreover, MS lesion or inactivation produces similar memory impairments to those observed after complete hippocampal lesions, suggesting that disruptions of MS activity have a secondary effect on hippocampal function (Donovick 1968; Numan 1978; Morris et al. 1982; Olton et al. 1982; Brookes et al. 1983; Rawlins et al. 1985; Kesner et al. 1989; Givens and Olton 1990; Bannerman et al. 2004). A key feature of SWM tasks is the delay period over which spatial information needs to be maintained. The inclusion of the delay period can affect the necessity of different brain systems for task performance. For example, complete hippocampal lesions were disruptive to performance of a T-maze delayed spatial alternation task with delays as short as $2 \mathrm{sec}$, but performance on the no-delay version of the task was unaffected by the lesion (Ainge et al. 2007), suggesting that the hippocampus is necessary for maintaining spatial information over a brief temporal gap. Based on previous work implicating the hippocampus in SWM maintenance over brief delays and knowing that the MS provides the hippocampus with critical input, our hypothesis was that

\section{Corresponding author: amygriff@udel.edu}

Article is online at http://www.learnmem.org/cgi/doi/10.1101/lm.053348. 120. the maintenance of spatial information over a temporal gap requires the MS. We tested this hypothesis by comparing the effects of optogenetic suppression of the MS between encoding, maintenance, and retrieval on choice accuracy of two different SWM-dependent tasks. The temporal precision of optogenetics allowed us to restrict MS suppression to specific periods within three different T-maze tasks (see Fig. 1). For experiment 1, we compared the effects of MS suppression on choice accuracy between two variants of a conditional discrimination task, in which rats learned to associate intramaze cues with a reward location. In the standard version of the task (CD), the conditional cue is available at the maze T-junction, where the rat is required to make a goal arm choice. Thus, the CD task does not require the maintenance of spatial information over a temporal delay. In the working memory dependent variant of the $\mathrm{CD}$ task (CDWM), the conditional cue is only available at the start of the trial, thus requiring the rat to maintain spatial information over a short temporal delay. We predicted that MS suppression would disrupt choice accuracy on the CDWM but not the CD variant of the task. We then compared the impact of MS suppression during the cue-sampling period and cue-retrieval period of the CDWM task. Based on the notion that the cue-retrieval period includes maintenance of the memory of the conditional cue and on our hypothesis that the MS is required for SWM maintenance, we predicted that there would be a selective deficit resulting from MS suppression during the cue-retrieval period. In experiment 2, we compared the effects of MS suppression that was selectively delivered during the sample, delay, and choice phases of a SWM-dependent delayed nonmatch to position

(C) 2021 Gemzik et al. This article is distributed exclusively by Cold Spring Harbor Laboratory Press for the first 12 months after the full-issue publication date (see http://learnmem.cshlp.org/site/misc/terms.xhtml). After 12 months, it is available under a Creative Commons License (Attribution-NonCommercial 4.0 International), as described at http://creativecommons.org/licenses/by-nc/ $4.0 \%$. 


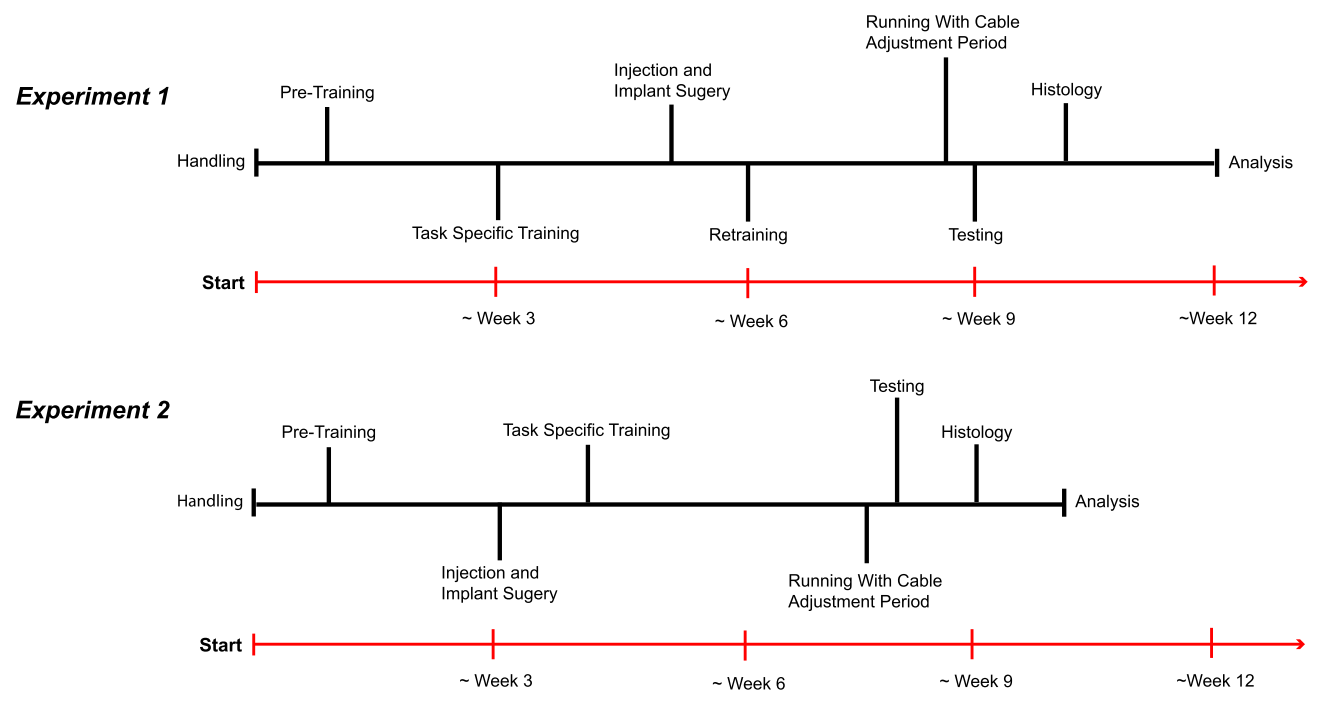

Figure 1. Experimental timeline for experiments 1 and 2.

(DNMP) task. Based on our hypothesis that MS is selectively required for SWM maintenance, we predicted that MS suppression during the delay, but not sample or choice phases, would impair DNMP choice accuracy.

\section{Results}

\section{Histology}

Figure 2A shows a representative image of tdTomato expression in the MS. We observed tdTomato expression throughout the MS and diagnoal band of Broca (DBB). A conceptual rendering of an optical fiber implant during illumnination is displayed in Figure 2B. Figure 2C shows the termination points of all fiber stubs. Rats with incorrect fiber stub placement and/or a lack of tdTomato expression in the MS/DBB were excluded from the study. For experiment 1 , there were nine rats in the $\mathrm{CDWM}+$ ArchT-tdTomato group, seven rats in the $\mathrm{CD}+$ ArchT-tdTomato, and seven rats in the CDWM+ tdTomato-only group. For experiment 2 , there were six rats in the ArchTtdTomato group and eight rats in the tdTomato-only group.

\section{Experiment 1: optogenetic silencing of MS impairs performance of the CDWM but not CD task}

We compared the ability of rats to perform two visuo-spatial conditional discrimination tasks during MS suppression: one that relies on SWM (CDWM) and one that does not (CD). In both tasks, floor inserts with either smooth wood or black mesh were placed in the maze prior to each trial, serving as a conditional cue for the rewarded goal arm. For the CD task, the insert covered the entire maze, including the T-junction, where the rats must make a goal arm choice. In contrast, for the CDWM task, the insert only covered the part of the maze stem immediately adjacent to the start box and was not present at the T-junction, requiring the rats to use working memory to make a goal arm choice (Edsall et al. 2017). Rats were trained on their respective tasks until they reached asymptotic choice accuracy. Rats took an average of $9.8 \mathrm{~d}(\mathrm{SD}=3.8 \mathrm{~d})$ to reach criterion on the CD task and $25.06 \mathrm{~d}$ $(\mathrm{SD}=7.2 \mathrm{~d})$ to reach criterion on the CDWM task.

We investigated the effect of optogenetic silencing of the MS on performance of the SWM-dependent CDWM task by comparing choice accuracy between Light-on versus Light-off in three

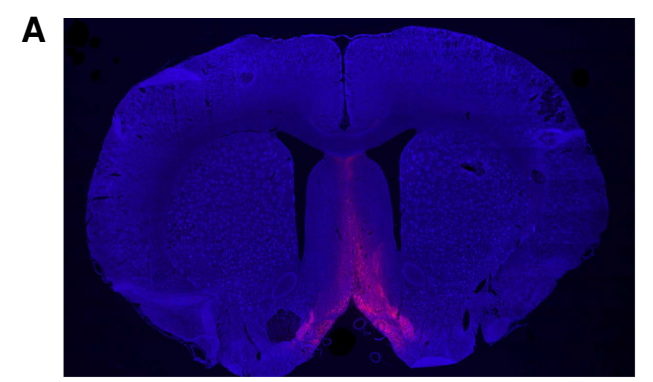

B

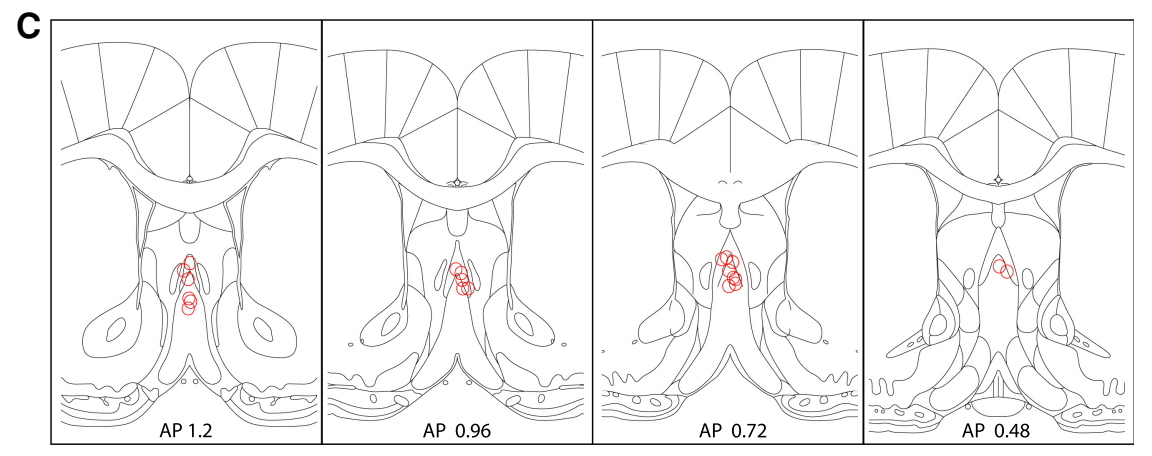

Figure 2. Histological verification of viral vector expression and optical fiber termination in MS. $(A)$ Representative expression of tdTomato in MS (tdTomato-positive cells are shown in pink and DAPI-positive cells are shown in blue). (B) Schematic of microinfusion and optogentic MS suppression procedures. (C) Sample coronal sections presented anterior to posterior at corresponding sections $(A P=+1.2$, $+0.96,+0.72$, and +0.48 , from bregma) showing fiber termination points for all animals in both experiments (red circles). Atlas plates are from Paxinos and Watson (2006), used with permission from Elsevier (C 2006). 
groups: a group that learned the SWM-independent CD task and were injected with the virus encoding the neural suppressor, ArchT (CD + ArchT-tdTomato; $n=7)$; a task control group that learned the SWM-dependent CD task and were injected with the virus encoding the ArchT (CDWM+ArchT-tdTomato; $n=9)$; and a virus control group that learned the CDWM task and were injected with a virus encoding only the reporter tdTomato (CDWM+ tdTomato-only; $n=7$ ). There were three illumination conditions, with MS suppression delivered during the entire trial, during the time when the rat occupied the portion of the maze where the conditional cue was available (early stem), and during the time when the rat occupied the portion of the maze where the conditional cue was absent (late stem).
A three-factor mixed design ANOVA showed a significant group $\times$ light $\times$ condition interaction $\left(F_{(1,13)}=11.798, P=0.004\right)$. In line with our predictions, post-hoc tests revealed a significant group $\times$ light interaction on the entire trial illumination condition $\left(F_{(1,14)}=18.520, P=0.0001\right)$ (Fig. 3A). However, in contrast to our predictions, there was a significant group $\times$ light interaction for the early stem illumination timing condition $\left(F_{(1,14)}=28.336\right.$, $P=0.001$ ) (Fig. 3B), but not the late stem illumination timing condition $\left(F_{(1,13)}=0.622, P=0.445\right)$ (Fig. 3C). For the late stem condition there were also no main effects of group $\left(F_{(1,13)}=0.041, P=0.445\right)$ or light $\left(F_{(1,13)}=1.377, P=0.262\right)$. For the entire trial illumination condition, the ArchT-tdTomato group showed a significantly lower choice accuracy on light on versus light off trials (Light-on:

A

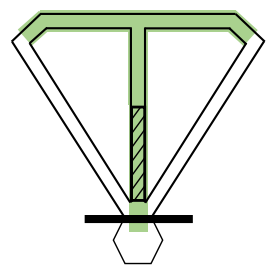

Entire

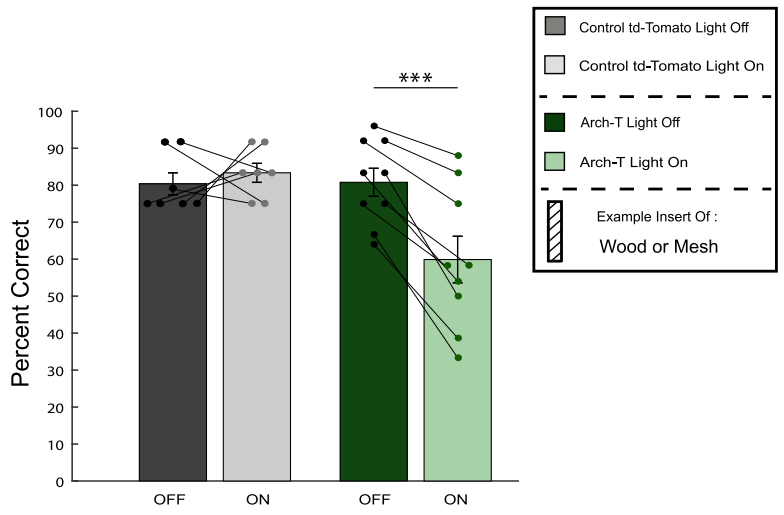

B

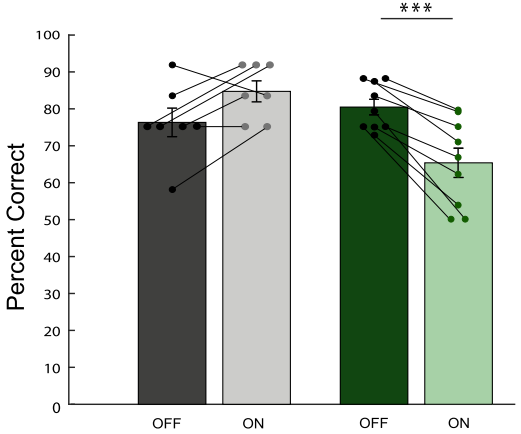

C

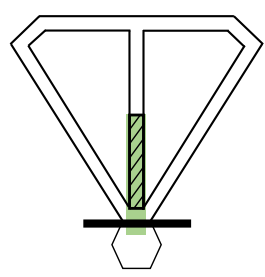

Early Stem

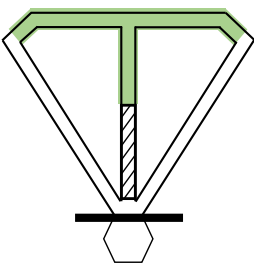

Late Stem

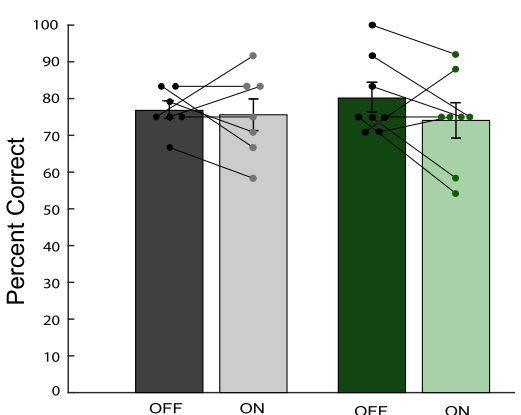

Figure 3. MS suppression impairs on choice accuracy on the CDWM task. ( $A-C$, left) Task schematics with green shading illustrating when the LED light was on for the entire, early, and late stem illumination conditions. The hashed rectangle represents the textured insert (wood or mesh) that was placed on the floor of the maze prior to each trial that served as the conditional cue. (Right) Choice accuracy is shown for the two groups: ArchT (green, $n=9$ ) and tdTomato control (gray, $n=7$ ). Significance values represent post-hoc Bonferroni-corrected simple main effects tests comparing Light-on versus Light-off trials. $\left.{ }^{* * *}\right) P<0.001$ (see the text for detailed statistics). $(A)$ Choice accuracy for the entire illumination condition. The ArchT group showed significantly lower choice accuracy on Light-on trials compared with Light-off trials, whereas the tdTomato control group showed similar choice accuracy values on Light-on and Light-off trials. (B) Choice accuracy for the early stem condition. The ArchT group showed significantly lower choice accuracy on Light-on trials versus Light-off trials, whereas the tdTomato group showed similar choice accuracy values on Light-on and Light-off trials. (C) Choice accuracy for the late stem illumination condition. Both ArchT and tdTomato groups showed similar choice accuracy values on Light-on and Light-off trials. (AC) Color legends are the same across all panels. Error bars indicate standard error of the mean. 
$M=59.9 \%, S D=0.178 ;$ Light-off: $M=81 \%, S D=0.107 ; t_{(8)}=6.39$, $P=0.0002$ ), while the tdTomato-only group showed similar choice accuracy levels across the Light-on and Light-off conditions (Light-on: $M=83.3 \%$, SD =0.063; Light-off: $M=80.0 \%$, SD $=0.073$; $\left.t_{(6)}=-0.611, P=0.563\right)$. For the early stem illumination condition, the ArchT-tdTomato group showed a significantly lower choice accuracy on light on versus light off trials (Light-on: $M=65.2 \%$, $\mathrm{SD}=0.112$; Light-off: $\left.M=80.3 \%, \mathrm{SD}=0.059 ; t_{(8)}=5.72, P=0.0004\right)$.

To confirm that choice accuracy deficits on CDWM were not simply caused by sensory, motor, or motivational impairments inherent to MS suppression during visuo-spatial conditional discrimination, we trained a separate group of rats expressing ArchT-tdTomato in the MS on the SWM-independent variant of the task (CD). As shown in Figure 4, MS suppression did not impair choice accuracy on the CD task (two-factor repeated measures ANOVA, light $\times$ condition interaction: $\left.F_{(1,6)}=0.832, P=0.397\right)$. There were no significant main effects of light $\left(F_{(1,6)}=0.519, P=\right.$ $0.498)$ or condition $\left(F_{(1,6)}=0.024, P=0.882\right)$.
In summary, experiment 1 showed that SWM depends on the integrity of the MS by showing impaired choice accuracy on the CDWM but not the CD task during optogenetic MS suppression. Moreover, the results of experiment 1 suggest that the timing of the MS suppression is important. In contrast to our predictions, MS suppression delivered during the time of conditional cue availability, but not during the time of conditional cue absence, caused a significant choice accuracy impairment.

\section{Experiment 2: MS suppression during the delay, but not} sample or choice conditions, impaired DNMP task accuracy To further investigate which phases of SWM MS activity supports, experiment 2 used a DNMP task, which is a task that has been widely used in experiments that aim to dissociate SWM encoding, maintenance, and retrieval processes (Kelsey and Landry 1988; Herzog et al. 1996; Stackman et al. 1997; Spellman et al. 2015; Bolkan et al. 2017; Maisson et al. 2018). By taking advantage of
A

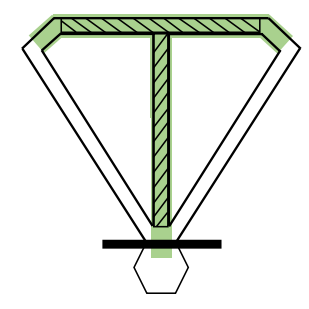

Entire

B

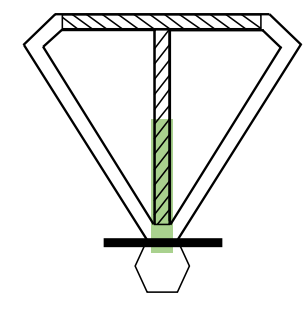

Early Stem

C

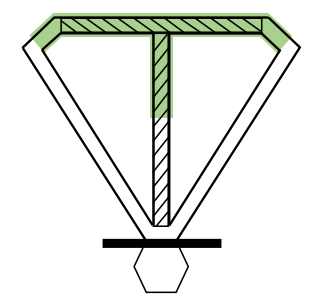

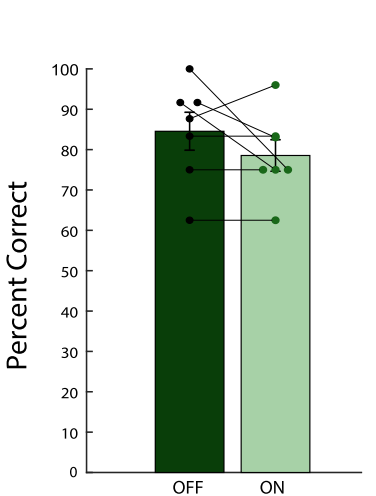
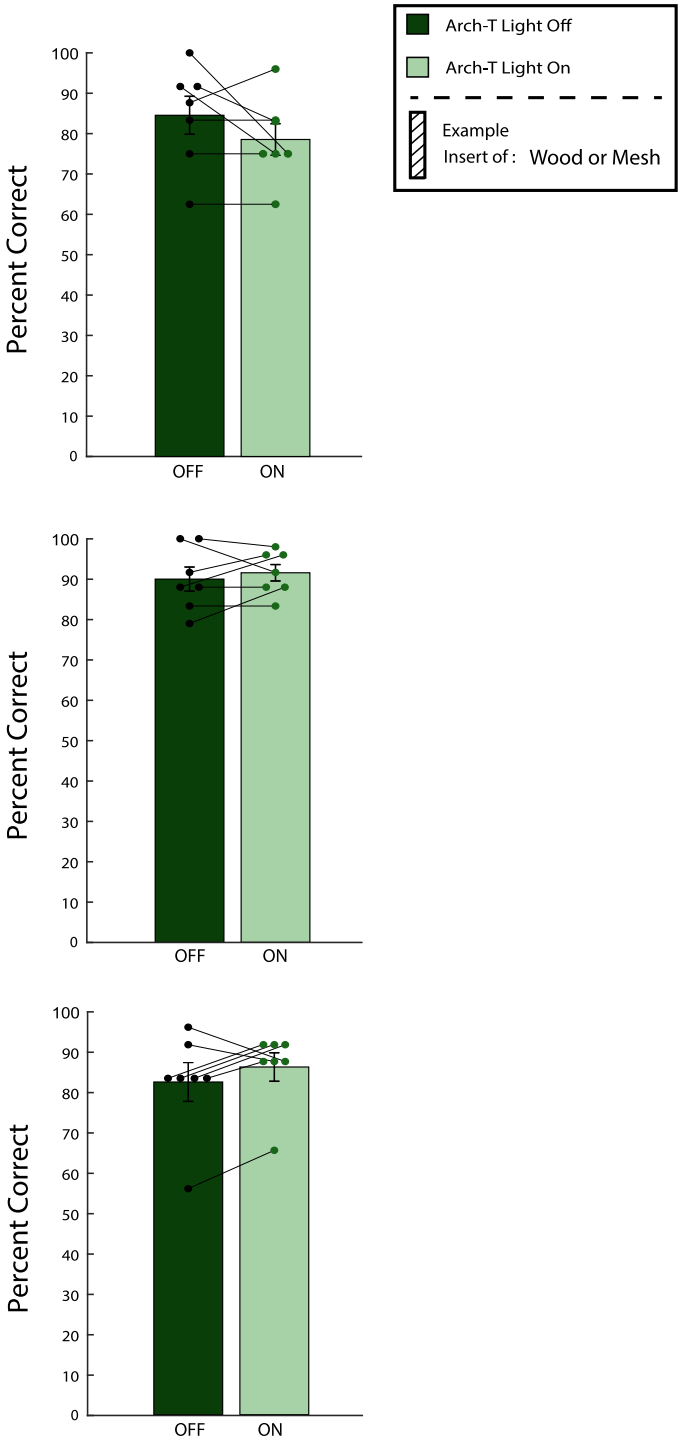

Figure 4. MS suppression does not impair on choice accuracy on the SWM-independent CD task. (A-C, left) Task schematics are as in Figure 3. Note that for this task, the floor insert covers the entire maze, including the maze T-junction. (Right) Choice accuracy is shown for the CD $+\operatorname{ArchT-tdTomato~group~(~} n$ $=7$ ) for the entire $(A)$, early stem $(B)$, and late stem $(C)$ illumination conditions. For all three conditions, the CD + ArchT-tdTomato group showed similar choice accuracy values on Light-on (dark green) and Light-off (light green) trials. 
the temporal precision of optogenetics, we silenced the MS specifically during the sample, delay, or choice phases of the DNMP task, which correspond to memory encoding, maintenance, and retrieval, respectively. Rats took an average of $5.6 \mathrm{~d}(\mathrm{SD}=2.2 \mathrm{~d})$ to reach criterion on the DNMP task. There was a significant light $\times$ group $\times$ condition interaction $\left(F_{(1,12)}=23.870, P<0.001\right)$. As shown in Figure $5 \mathrm{~A}$, both ArchT-tdTomato and tdTomato-only groups showed similar levels of choice accuracy for Light-on and Light-off trials during the sample illumination condition (mixed design two-factor ANOVA with repeated measures, no main effect of group: $F_{(1,12)}=0.640, P=0.439$, group $\times$ light interaction: $F_{(1,12)}=$ $0.054, P=0.821$ ). In contrast, for the delay illumination condition, a mixed design two-factor ANOVA revealed a significant group $\times$ light interaction $\left(F_{(1,12)}=5.385, P=0.039\right)$. Bonferroni-corrected post-hoc tests revealed that the ArchT-tdTomato group performed more poorly on Light-on trials $(\mathrm{M}=74.0 \%, \mathrm{SD}=0.132)$ compared with Light-off trials $\left(\mathrm{M}=84.7 \%, \mathrm{SD}=0.131 ; t_{(5)}=3.369, p=0.02\right)$. The tdTomato-only group performed similarly during Light-on trials $(\mathrm{M}=88.5 \%, \mathrm{SD}=0.0797)$ and Light-off trials $(\mathrm{M}=90.6 \%$, $\mathrm{SD}=0.048 ; t_{(7)}=-0.509, P=0.626$ ) (Fig. $\left.5 \mathrm{~B}\right)$. For the choice phase condition (Fig. 5C), a two-way mixed design repeated measures ANOVA showed a trending group by light interaction. However, the interaction did not reach statistical significance $\left(F_{(1,12)}=\right.$ 3.432, $P=0.089)$. Moreover, there were no significant main effects of group $\left(F_{(1,12)}=0.456, P=0.512\right)$ or light $\left(F_{(1,12)}=1.415\right.$, $P=0.257)$ for the choice phase condition. In summary, we found a selective impairment in choice accuracy that resulted from MS suppression during the delay phase, suggesting that the integrity of the MS is critical for SWM maintenance.

\section{Discussion}

SWM requires the encoding of sensory cues that must be retrieved after a temporal gap in order to guide goal-directed decision making. In the current study, we tested the hypothesis that the MS is

A

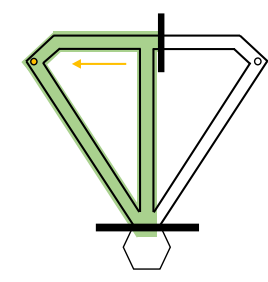

Sample Phase
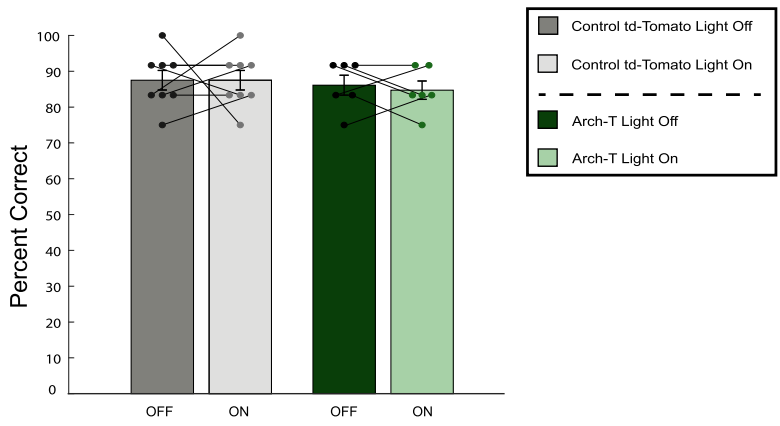

B

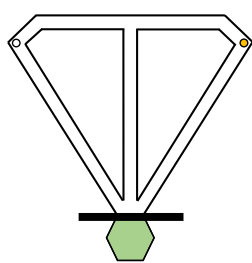

Delay Phase

( 20 Seconds)

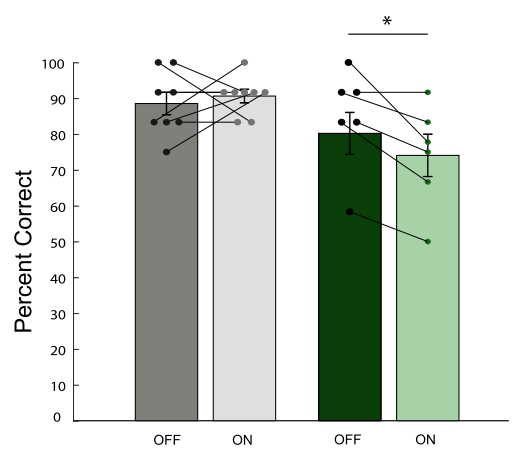

C

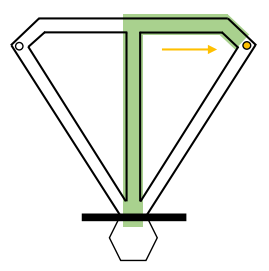

Choice Phase

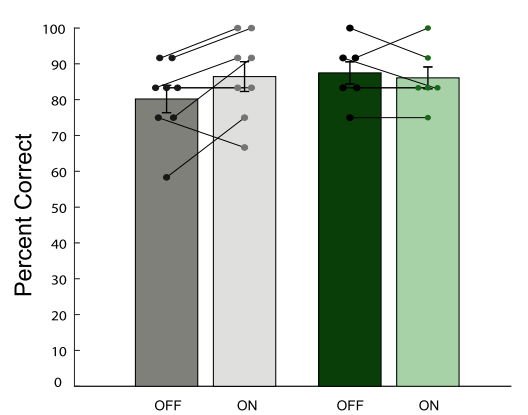

Figure 5. MS suppression selectively impairs DNMP task choice accuracy during SWM maintenance. ( $A-C$, left) Task schematics with green shading illustrating when the LED light was on for the sample, delay, or choice phase testing conditions. (Right) Choice accuracy is shown for the two groups: ArchT (green, $n=6)$ and tdTomato control (gray, $n=8$ ). Significance values represent post-hoc Bonferroni-corrected simple main effects tests comparing Light-on versus Light-off trials. $\left(^{*}\right) P<0.05$ (see the text for detailed statistics). (A) Choice accuracy for the sample phase illumination condition. Both ArchT and tdTomato groups showed similar choice accuracy values on Light-on and Light-off trials. (B) Choice accuracy for the delay illumination condition. The ArchT group showed significantly lower choice accuracy on Light-on trials versus Light-off trials, whereas the tdTomato group showed similar choice accuracy values on Light-on and Light-off trials. (C) Choice accuracy for the choice phase illumination condition. Both ArchT and tdTomato groups showed similar choice accuracy values on Light-on and Light-off trials. 
required for maintenance of spatial information over a temporal delay by comparing the effects of pan-neuronal optogenetic suppression of MS activity on the performance of three T-maze tasks, two of which required the use of SWM processes and one that was SWM-independent. In experiment 1 , we found that MS optogenetic suppression impaired choice accuracy on the SWM-dependent CDWM task, but not the SWM-independent CD task. Because these two tasks are alike in every respect except their reliance on SWM, this dissociation allowed us to rule out the possibility that our findings are driven by deficits in motor, sensory, motivational, or other nonmnemonic variables. Additionally, we restricted MS suppression to the early and late stem portions of the CD and CDWM tasks. For the CDWM task, MS suppression on the early stem, when the floor insert cue was present, impaired choice accuracy while MS suppression during the late stem condition on the CDWM task, when the cue was absent, did not. In contrast, for the CD task, we found no choice accuracy deficits when MS suppression was delivered on the entire trial, or during early or late stem. For experiment 2, we restricted MS suppression to the sample, delay, or choice phases of a DNMP task. We found that MS suppression during the delay phase, but not sample or choice phases, impaired choice accuracy.

The results from these experiments are consistent with previous research showing the importance of the MS in SWM tasks (Olton et al. 1978; Hagan et al. 1988; Kelsey and Landry 1988; Decker et al. 1992; Bolding et al. 2020; Petersen and Buzsaki 2020). However, our results expand on these studies by showing that MS inhibition during the period of time over which spatial information must be maintained specifically impairs choice accuracy. Collectively, our findings support the hypothesis that SWM maintenance requires the MS.

One puzzling previous result that conflicts with our current findings is from a study using septal inactivation during radial maze performance in rats. Similar to the DNMP task, each trial in this task consisted of forced choices (of four goal arms), a delay period, and a test allowing the rat to freely choose a goal arm, but only rewarding those arms not visited previously. Septal inactivation prior to both the forced choice phase of the task and the test phase of the task increased errors during the test phase. In contrast, septal inactivation prior to the delay period did not affect task performance (Mizumori et al. 1990). One key difference between this study and our study is that the delay period in the previous study was $30 \mathrm{~min}$, whereas our delay period was only $20 \mathrm{sec}$. Perhaps the maintenance of spatial information is more feasible over a relatively short delay period versus a long delay period. If this is the case, the radial arm maze task would not require SWM maintenance and instead relies on another set of cognitive operations also supported by the MS.

A potential limitation of experiment 2 is that the duration of the optogenetic suppression was longer for the delay period than for the sample or choice traversals. Thus, there is a possibility that the longer duration of MS suppression drove the selective disruption of choice accuracy on the DNMP task during the delay period illumination condition. However, previous studies have used a similar approach of delivering optogenetic suppression during the sample traversals, delay period, and choice traversals of a DNMP task and have shown varying results depending on the brain region or pathway that was targeted. A previous study from our laboratory investigated the effects of optogenetic suppression of the ventral midline thalamus on encoding, maintenance, and retrieval in a DNMP task and found a selective choice accuracy deficit on the sample traversal illumination condition and no deficit on the delay period illumination condition (Maisson et al. 2018). Similarly, optogenetic suppression of the ventral hippocampus-prefrontal pathway led to deficits only when the light delivery was restricted to the sample traversals and not to the delay or choice traversals of a DNMP task (Spellman et al. 2015). Moreover, optogenetic suppression of the pathway between the mediodorsal (MD) thalamus and prefrontal cortex also showed different results depending on the specific pathway that was suppressed, with DNMP choice accuracy being selectively impaired with suppression of MD-prefrontal projections during the delay and with suppression of prefrontalMD projections during choice traversals (Bolkan et al. 2017). The results of experiment 1 also argue against the possibility that longer-duration optogenetic suppression can explain our selective choice accuracy deficit on the delay period illumination condition. The early stem and late stem illumination conditions had similar durations, but deficits were only observed with MS suppression on the early stem. If longer-duration optogenetic stimulation leads to a choice accuracy deficit, we would predict that longer-duration optogenetic stimulation would consistently produce selective choice accuracy impairments. Therefore, these results collectively suggest that it is unlikely that the delay period-specific effect in experiment 2 was driven by the duration of MS suppression.

In both the CDWM and DNMP task, there is a temporal gap between when rats must encode a cue and when they must retrieve the cue to guide their choice. Conversely, no such gap exists in the CD task, as the rat is able to sample the cue during decision making. The fact that both CDWM and DNMP tasks are impaired with MS suppression supports the hypothesis that the MS contributes to the maintenance of spatial information over a brief temporal gap. Further supporting this hypothesis, the choice accuracy deficit on the DNMP task was only observed when the MS suppression was delivered during the delay. Compared with the DNMP task, for the CDWM task, there is a much shorter time from when the rats must encode the cue on the early stem portion and retrieve the cue and make a decision at the T-junction of the same traversal. In opposition to our predictions, MS suppression in the early stem condition but not in the late stem condition resulted in a choice accuracy deficit. Although our prediction was incorrect, the choice accuracy deficit for MS suppression delivered during the cuesampling period is an interesting finding and suggests that perhaps maintenance and encoding processes may overlap in tasks that lack a structured delay period. This possibility could be investigated by inserting a delay between the cue-sampling period and the cue-retrieval period of the CDWM task to better dissociate encoding and maintenance processes.

Why might MS disruption selectively impair the maintenance and not encoding or retrieval of SWM? One possibility is that MS suppression indirectly affected the projection regions of the MS, including the hippocampus and medial entorhinal cortex (MEC). One well-established function of the MS is to act as a pacemaker for the hippocampal theta rhythm (Stewart and Fox 1990). Support for this notion comes from the demonstrations that the frequency of the hippocampal local field potential could be controlled by optogenetically stimulating GABAergic (Zutshi et al. 2018), cholinergic (Dannenberg et al. 2015), and glutamatergic (Robinson et al. 2016) MS neurons. Although we did not record hippocampal theta activity in the current study, previous work showing consistent disruptions of the theta rhythm within the hippocampal/MEC circuit as a consequence of MS lesions (Donovick 1968; Numan 1978; Winson 1978), reversible inactivation (Mizumori et al. 1990; Wang et al. 2015), or optogenetic suppression of the MS (Dannenberg et al. 2019) suggests that the behavioral impairments that we observed may be due to the disruption of theta activity. Recent work has shown that microinfusions of gabazine into the MS, which disrupted normal hippocampal oscillatory activity, caused SWM deficits while the spatial tuning of hippocampal neurons was unaffected by the drug (Bolding et al. 2020). This finding suggests that MS-driven SWM deficits are caused by the loss of the theta rhythm itself, not by a secondary effect of impaired theta rhythm on the spatial 
specificity of these neurons. MS inactivation was also shown to disrupt grid cell spatial periodicity in the MEC without affecting the head direction signal, supporting the notion that MS-driven theta oscillations support spatial coding in the MEC (Brandon et al. 2011; Koenig et al. 2011).

In summary, by using a task comparison optogenetic suppression approach, we show that the MS is required for tasks that require SWM and not for a SWM-independent task. Furthermore, by comparing the timing of MS suppression between different portions of the trial, we show that the MS specifically contributes to the maintenance of spatial information over a brief delay. This contribution likely arises from the important role that the MS plays in regulating activity in the hippocampus and related structures.

\section{Materials and Methods}

\section{Subjects}

Subjects were 37 adult ( $>90$ d) Long Evans hooded male rats purchased from Envigo or Harlan. Rats were housed in a temperature and humidity-controlled colony room with a 12-h light-dark cycle. At the onset of the experiment, rats were single housed and given ad libitum access to food until pretraining began. At the start of pretraining, rats were placed on light food restriction and kept at a minimum $90 \%$ of their ad libitum body weight. Rats had ad libitum access to water throughout the experiment. All procedures in this experiment were conducted in accordance with the University of Delaware Institutional Animal Care and Use Committee.

\section{Apparatus}

Testing took place in an enclosed rectangular room (2.29 $\mathrm{m}$ wide $\times$ $2.46 \mathrm{~m}$ long) with black curtains spanning from floor to ceiling. Visual cues were placed on the curtains with tape behind the goal arms and the T-junction of a T-maze. The wooden T-maze used was painted flat white to aid in reduction of reflections. The T-maze consisted of a central arm $(116 \times 10 \mathrm{~cm})$, two goal arms $(56.5 \times 10 \mathrm{~cm})$, and two return arms leading back to the start box/delay pedestal $(112 \times 10 \mathrm{~cm})$. To reduce the probability that rats could follow scent cues to solve the task, a container filled with chocolate sprinkles was secured beneath the T-junction of the maze, out of reach by the rats. The testing enclosure was dimly lit with two 1000-lumen, $120-\mathrm{V}, 60-\mathrm{Hz}$ LED bulbs located above the reward wells on both sides of the maze.

Optical LED stimulation was made possible by the PlexBright optogenetic stimulation system (Plexon), which consisted of a four-channel optogenetic controller with installed Radiant v2 software, a commutator, and a compact magnetic LED "lime" colored module $(550 \mathrm{~nm})$ that produced a maximum power output of $\sim 12$ $\mathrm{mW}$. The LED light was channeled into the rat's fiber stub via a patch cable and secured with a removable ceramic coupler (Thorlabs ADAF1-5).

\section{Handling and pretraining}

Rats were handled for at least $20 \mathrm{~min}$ per day for at least $3 \mathrm{~d}$ prior to and at least $5 \mathrm{~d}$ after the surgery to habituate them to the experimenter. After each handling session, the rats were returned to their home cage with a small weigh boat filled with chocolate sprinkles to help familiarize the rats with the food reward used during task training. After $5 \mathrm{~d}$ of postsurgical recovery, rats were placed on food restriction ( $\sim 17 \mathrm{~g}$ of standard rat chow/day) in order to maintain $90 \%$ of their ad libitum body weight. The rats were then pretrained to eat the chocolate sprinkle reward from the reward wells while confined to one of the maze reward zones, as described previously (Hallock et al. 2013b; Maisson et al. 2018; Stout and Griffin 2020). Following reward well pretraining, rats were pretrained to run from the start box to the reward zones via the maze stem. Each of these "forced run" trials began with the rat confined in the start box by a barrier that blocked the entrance to the maze stem. Prior to each trial, the reward wells were baited with the chocolate sprinkles and a barrier was placed at the entrance to the right or left goal arm. The stem barrier was then lifted, allowing the rat to traverse the stem of the maze and enter the open goal arm. After the rat received the reward, the rat returned to the start box via the return arm. The stem barrier was then replaced, and the reward wells are baited again for the next trial. Rats completed 12 forced run trials (six left trials and six right trials) per day until they reached the necessary criterion of consuming reward on every trial.

\section{Surgical procedures}

Prior to surgery, rats were given a subcutaneous injection of atropine $(0.05 \mathrm{mg} / \mathrm{kg}$; Atroject). All instruments were sterilized using a bead sterilizer at $300^{\circ} \mathrm{C}$ and cleaned in chlorhexidine solution. Rats were anesthetized using 3.5\% isoflurane in oxygen in a Plexiglass induction chamber. Once anesthetized, ophthalmic lubricant (Paralube) was applied to eyes and the rats' heads were shaved at the incision site. For the duration of the surgery, rats were head-fixed in a stereotaxic frame to ensure continuous flow of isoflurane in oxygen. The rats were placed on top of a heating pad throughout the surgery and during recovery. The rats' breathing, heart rate, and oxygen levels were monitored throughout surgery using a pulse oximeter via foot clamp. Paralube ophthalmic lubricant was reapplied before the incision site was cleaned using chlorhexidine solution. A subcutaneous injection of lidocaine (4 $\mathrm{mg} / \mathrm{kg}$ ) was given prior to the incision. The skull was cleaned and leveled by comparing the dorsal-ventral (DV) coordinates of bregma and lambda. Once leveled, a bregma measurement was taken to determine the anterior-posterior (AP) and medial-lateral (ML) coordinates for virus injection and fiber implantation. Four holes were drilled into the skull using a stereotaxic mounted drill (Foredom HP4-917 control unit with H.MH-170 handpiece) and self-tapping bone screws were inserted (Fine Science Tools 19010-00, 1.19-mm diameter by 4.8-mm length) to help anchor the implant to skull.

\section{Viral constructs}

This study used two recombinant adeno-associated viral (AAV) vector constructs purchased from University of North Carolina Vector Core. The control virus expressed the red fluorescent protein tdTomato alone (AAV5-CAG-tdTomato), and the neural silencer expressed an archaerhodopsin-tdTomato fusion (AAV5-CAGArchT-tdTomato). Both constructs contained the CAG promoter and were constructed of titer $3.1 \times 10^{12}$ virus molecules $/ \mathrm{mL}$.

\section{Viral vector microinjections}

Experiments 1 and 2 used slightly different surgical coordinates in order to target the MS; however, histology confirmed that viral spread in the MS was comparable across both experiments. A trephine (Meisinger Company) was used to make a single craniotomy over the MS (experiment 1: $\mathrm{AP}+0.7 \mathrm{~mm}$, ML $0 \mathrm{~mm}$ in reference to bregma; experiment 2: $\mathrm{AP}+0.7 \mathrm{~mm}, \mathrm{ML}+1.1 \mathrm{~mm})$. Next, an absorbable gelatin sponge was soaked in sterile saline and applied to the exposed tissue to prevent drying. All injections were performed using a Hamilton syringe attached to a Pump 11 Elite Nanomite pump and Nanomite injector unit (Harvard Apparatus 70-4507, serial no. D-301251). The viral vector construct was microinjected into the MS at multiple coordinates in both experiments with anterior-posterior (AP) and medial-lateral (ML) coordinates referenced to bregma and dorsal-ventral (DV) coordinates referenced to dura. For experiment 1, there were four infusions of $0.25 \mu \mathrm{L}$ of each at $\mathrm{AP}=+0.7 \mathrm{~mm}, \mathrm{ML}=0.0 \mathrm{~mm}, \mathrm{DV}=$ $-6.5 \mathrm{~mm},-6.3 \mathrm{~mm},-6.0 \mathrm{~mm},-5.8 \mathrm{~mm}$ ). For experiment 2 , two infusions containing $0.5 \mu \mathrm{L}$ of each were performed at a $10^{\circ}$ angle lateral to the midline $(\mathrm{AP}=+0.7 \mathrm{~mm}, \mathrm{ML}=+1.1 \mathrm{~mm}, \mathrm{DV}=-6 \mathrm{~mm}$, $-5.5 \mathrm{~mm}$ ). For each coordinate, the needle was lowered to the more ventral site first and five minutes was allotted to allow tissue to settle around the needle before injection. The viral vector construct was injected via a neuros syringe (Hamilton Co.) at rate of $0.1 \mu \mathrm{L} / \mathrm{min}$ (flow rate at $70 \%$ ). The needle was then raised to the 
more dorsal site, and the virus was infused. Following the final injection, the needle was left in place for $10 \mathrm{~min}$ to allow the virus solution to diffuse into the tissue. Saline soaked Vetspon was placed over the craniotomy to prevent the brain surface from drying out. GLUture was then used to fill the craniotomy immediately following the fiber stub implant.

\section{Optogenetic fiber implantation}

Prefabricated fiber stubs (Plexon 200/230- $\mu \mathrm{m}$ fiber, ferrule tip length $2.5 \mathrm{~mm}$, total stub length $10 \mathrm{~mm}$ ) were manually scored with a diamond wheel dremel bit and implanted targeting the MS (experiment $1: \mathrm{AP}=+0.7 \mathrm{~mm}$ and $\mathrm{ML}=0.0 \mathrm{~mm}$ from bregma, $\mathrm{DV}=-5.5 \mathrm{~mm}$ from dura; experiment 2 [implanted at a $10^{\circ}$ angle]: $\mathrm{AP}=+0.7$ and $\mathrm{ML}=+1.1$ from bregma, $\mathrm{DV}=-5.3$ from dura). GLUture was then used to cover the craniotomy and allowed to dry. The exposed skull was allowed to dry for several minutes to ensure proper adhesion of the dental acrylic. A small dome of dental acrylic (Lang Dental) was built up to encapsulate the bottom of the scored fiber and bone screws were added to anchor the implant to the skull.

\section{$\mathrm{CD}$ and $\mathrm{CDWM}$ task training (experiment 1 )}

We trained separate groups of rats to perform variants of a visuo-spatial conditional discrimination task, one that is SWM-independent and one that is SWM-dependent (CD and CDWM, respectively). These task variants have been described previously (Griffin et al. 2012; Hallock and Griffin 2013; Hallock et al. 2013a,b; Shaw et al. 2013; Edsall et al. 2017). Briefly, for the CDWM task, a wooden floor insert covered with black mesh on one side and the other side bare wood, was placed in the bottom half of the stem prior to each trial. Each rat was randomly assigned a conditional rule (left on mesh, right on wood, or vice versa) that remained consistent throughout the experiment. Prior to each trial, the left or right reward well was filled with chocolate sprinkles so that rats only earned a reward when they followed the rule. Each training session consisted of 24 trials (12 wood and 12 mesh trials) presented in a pseudorandom sequence with a 20 -sec intertrial interval (ITI). Rats were required to meet the choice accuracy criterion of $80 \%$ correct for two consecutive days before they were used for the optogenetic MS suppression stage of the experiment.

For the CD variant of the task, the floor insert spanned the entire maze stem, including the T-junction. The group of rats trained on CDWM variant of the task first learned the CD variant. After reaching criterion on the $\mathrm{CD}$ task, progressively shorter inserts were used each day until the inserts spanned from the stem entrance to halfway up the stem.

\section{Delayed nonmatch to position (DNMP) task training (experiment 2)}

The delayed nonmatched to position (DNMP) task consisted of a sample phase, a delay phase, and choice phase. Previous work from our laboratory and others has used the DNMP task to restrict optogenetic manipulations to these specific phases (Spellman et al. 2015; Bolkan et al. 2017; Maisson et al. 2018). Before each trial, the rat was confined to the start box with a barrier, reward wells on both arms of the T-maze are baited, and a barrier was placed at the entrance of either the right or left goal arm. To begin the sample phase, the barrier between the start box and the stem was lifted, allowing the rat to traverse the stem, enter the open goal arm, and consume the reward at the end of the goal arm. The rat then returned to the start box via the return arm and the stem barrier was returned for the 20 -sec delay, during which time the experimenter removed the goal arm barrier. The choice phase then began when the stem barrier was lifted, allowing the rat to reenter the maze stem and approach the T-junction. The rat then had a free choice to turn into the left or right goal arm. Rats earned a reward by selecting the opposite goal arm from the arm visited on the sample traversal. Rats then returned to the start box once again, where they were confined for the 40 -sec ITI. Each training session consisted of 24 trials (12 left and 12 right trials), presented in a pseudoran- dom sequence. Rats were required to meet the choice accuracy criterion of $80 \%$ correct for two consecutive days before they were used for the optogenetic MS suppression stage of the experiment.

\section{Optogenetic MS suppression during CDWM and $C D$ (experiment 1 )}

After meeting the behavioral task criterion, each rat underwent three acclimation sessions with the patch cable attached but no light stimulation delivered. This was done to ensure that having the patch cable attached to the head stage did not interfere with task performance during optogenetic MS suppression sessions. Before each testing session, the power output at the tip of the fiber attached to the patch cable was tested using a power meter (Thorlabs) to make sure that the power output was sufficient for optogenetic suppression. A minimum LED power output of $7 \mathrm{~mW}$ was used as the power threshold and each day of testing power ranged from 7 to $12 \mathrm{~mW}$. The rat was brought into the testing room and placed in the start box. Then both the fiber stub on the rat's head stage and the tether cable fiber were cleaned with optic fiber cleaner before they were connected via a ceramic coupler (Thorlabs) and the CD or CDWM testing session started. Both tasks were comprised of three light conditions: entire, early stem, and late stem conditions (Figs. 1, 3, 4). For CDWM task early stem condition, the light was turned on when the barrier between the start box and center arm was lifted and turned off after the rat's hind legs left the insert cue halfway up the stem. In the CD task, the light was turned off at the same spot on the maze, although the insert cue used in this task continued up the rest of the stem. For the late stem condition, the light stimulation started after the rat's hind legs left the insert in the CDWM task or in the equivalent place in the CD task and terminated right before the reward well. In the entire condition, light stimulation began as the barrier was lifted and continued until the rat reached the reward well. For all illumination conditions, the light was switched on and off by the experimenter. Seven rats completed at least one session of the each testing condition, while 30 rats completed two testing sessions. For rats that completed two testing sessions, choice accuracy scores were averaged. Testing sessions consisted of 24 trials: 12 Light-off trials and 12 Light-on trials presented in a pseudorandom order. The testing sessions for entire, early stem, and late stem conditions were counter balanced across rats.

\section{Optogenetic suppression of MS during DNMP (experiment 2)}

Once rats were able to consistently perform the DNMP task (two consecutive days at $80 \%$ choice accuracy or higher), they moved on to the behavioral testing portion of the experiment. Similar to experiment 1 , each rat underwent three patch cable acclimation sessions and the power output of the patch cable was tested prior to each session. Three conditions were then administered on separate sessions: illumination restricted to sample traversals, the delay, and choice traversals. For the sample condition, the LED was turned on as the barrier was lifted to start the sample traversal and terminated when the rat entered the start box. For the delay condition, the light was on for the 20 -sec delay between sample and choice traversals. For the choice condition, the light was turned on as the barrier was lifted to start the choice phase and terminated when the rat entered one of the goal arms. Like experiment 1 , for all illumination conditions, the light was switched on and off by the experimenter. Each session consisted of 24 trials, 12 Light-on and 12 Light-off, presented in a pseudorandom order. The order of the illumination condition sessions was counterbalanced across rats.

\section{Histological procedures}

At the conclusion of the behavioral testing portion of the experiment, rats were anesthetized at 3.5\%-4\% Isoflurane in oxygen and closely observed for motor responses to a toe pinch and eye blink reaction. Once fully anesthetized, a lethal dose of sodium 
pentobarbital was injected and the rat was perfused transcardially with $200 \mathrm{~mL}$ of phosphate-buffered saline (PBS) followed by 200 $\mathrm{mL}$ of $4 \%$ paraformaldehyde (PFA) in PBS. Upon successful perfusion, brains were immediately extracted and stored in 4\% PFA for $24 \mathrm{~h}$ and then switched over to a 30\% sucrose solution for cryoprotection. The brains were then mounted using Tissue-Tek (Fisher) and sliced at $-20^{\circ} \mathrm{C}$ on a Lieca $3050 \mathrm{~S}$ cryostat into $20-\mu \mathrm{m}$ slices and mounted on slides. The slides were washed three times for 5 min each in $1 \times$ PBS for cleaning. Next, slides were stained for cell bodies by applying Prolong Diamond containing 4',6-diamidino2-phenylindole (DAPI; Thermo Fisher Scientific), which is used to help visualize cell bodies by staining nuclear DNA (Tarnowski et al. 1991). The slides were then coverslipped and generic clear "quick-dry" nail polish was used to seal the coverslips to slides. The sections were imaged using a LSM 710 and 880 confocal microscope (Zen 2012 SP5 software) under a 10×/0.3 objective.

\section{Data analysis}

Choice accuracy on the behavioral tasks was compared between groups and light conditions with a three-way mixed factor ANOVA with repeated measures, with viral vector (ArchTtdTomato vs. tdTomato-only) as a between-subjects factor and light (on vs. off) and illumination timing condition (DNMP: sample, delay, or choice; CDWM: entire trial, early stem, or late stem) as within-subjects factors. For the task control group in experiment 1 , choice accuracy on the CD task was analyzed using a light (on vs. off) $\times$ illumination timing (early stem, late stem, or entire trial) repeated measures ANOVA. All post-hoc tests used Bonferroni corrections to correct for multiple comparisons.

\section{Competing interest statement}

The authors declare no competing interests.

\section{Acknowledgments}

This work was supported by National Institutes of Health (NIH) R01 MH102394. We thank Sami Weinstein, Camryn Isaksen, John Stout, and Andrew Garcia for technical support. We also thank John Stout for comments on the manuscript. Microscopy access was supported by grants from the NIH-National Institute of General Medical Sciences (P20 GM103446), the National Science Foundation (IIA-1301765), and the state of Delaware.

Author contributions: The study was designed by Z.M.G., M.M. D, and A.L.G. Z.M.G., and M.M.D. collected and analyzed the data. Z.M.G., M.M.D., and A.L.G. wrote the manuscript.

\section{References}

Ainge JA, van der Meer MA, Langston RF, Wood ER. 2007. Exploring the role of context-dependent hippocampal activity in spatial alternation behavior. Hippocampus 17: 988-1002. doi:10.1002/hipo.20301

Bannerman DM, Matthews P, Deacon RM, Rawlins JN. 2004. Medial septal lesions mimic effects of both selective dorsal and ventral hippocampal lesions. Behav Neurosci 118: 1033-1041. doi:10.1037/0735-7044.118.5 .1033

Bolding KA, Ferbinteanu J, Fox SE, Muller RU. 2020. Place cell firing cannot support navigation without intact septal circuits. Hippocampus 30: 175191. doi:10.1002/hipo.23136

Bolkan SS, Stujenske JM, Parnaudeau S, Spellman TJ, Rauffenbart C, Abbas AI, Harris AZ, Gordon JA, Kellendonk C. 2017. Thalamic projections sustain prefrontal activity during working memory maintenance. Nat Neurosci 20: 987-996. doi:10.1038/nn.4568

Brandon MP, Bogaard AR, Libby CP, Connerney MA, Gupta K, Hasselmo ME. 2011. Reduction of theta rhythm dissociates grid cell spatial periodicity from directional tuning. Science 332: 595-599. doi:10 $.1126 /$ science. 1201652

Brookes S, Rawlins JNP, Gray JA, Feldon J. 1983. DRL performance in rats with medial or lateral septal lesions. Physiol Psychol 11: 178-184. doi:10 .3758/BF03326791

Dannenberg H, Pabst M, Braganza O, Schoch S, Niediek J, Bayraktar M, Mormann F, Beck H. 2015. Synergy of direct and indirect cholinergic septo-hippocampal pathways coordinates firing in hippocampal networks. J Neurosci 35: 8394-8410. doi:10.1523/JNEUROSCI.4460-14 .2015

Dannenberg H, Kelley C, Hoyland A, Monaghan CK, Hasselmo ME. 2019 The firing rate speed code of entorhinal speed cells differs across behaviorally relevant time scales and does not depend on medial septum inputs. J Neurosci 39: 3434-3453. doi:10.1523/JNEUROSCI.1450-18 .2019

Decker MW, Majchrzak MJ, Anderson DJ. 1992. Effects of nicotine on spatial memory deficits in rats with septal lesions. Brain Res 572: 281-285. doi:10.1016/0006-8993(92)90485-r

Donovick PJ. 1968. Effects of localized septal lesions on hippocampal EEG activity and behavior in rats. J Comp Physiol Psychol 66: 569-578. doi:10 $.1037 / \mathrm{h} 0026514$

Edsall A, Gemzik Z, Griffin A. 2017. A tactile-visual conditional discrimination task for testing spatial working memory in rats. Bio Protoc 7: e2282. doi:10.21769/BioProtoc.2282

Freund TF, Antal M. 1988. GABA-containing neurons in the septum control inhibitory interneurons in the hippocampus. Nature 336: 170-173. doi: $10.1038 / 336170 \mathrm{a} 0$

Frotscher M, Leranth C. 1985. Cholinergic innervation of the rat hippocampus as revealed by choline acetyltransferase immunocytochemistry: a combined light and electron microscopic study. J Comp Neurol 239: 237-246. doi:10.1002/cne.902390210

Fuhrmann F, Justus D, Sosulina L, Kaneko H, Beutel T, Friedrichs D, Schoch S, Schwarz MK, Fuhrmann M, Remy S. 2015. Locomotion, theta oscillations, and the speed-correlated firing of hippocampal neurons are controlled by a medial septal glutamatergic circuit. Neuron 86: 12531264. doi:10.1016/j.neuron.2015.05.001

Givens BS, Olton DS. 1990. Cholinergic and GABAergic modulation of medial septal area: effect on working memory. Behav Neurosci 104: 849855. doi:10.1037//0735-7044.104.6.849

Griffin AL, Owens CB, Peters GJ, Adelman PC, Cline KM. 2012. Spatial representations in dorsal hippocampal neurons during a tactile-visual conditional discrimination task. Hippocampus 22: 299-308. doi:10 .1002/hipo.20898

Hagan JJ, Salamone JD, Simpson J, Iversen SD, Morris RG. 1988. Place navigation in rats is impaired by lesions of medial septum and diagonal band but not nucleus basalis magnocellularis. Behav Brain Res 27: 9-20. doi:10.1016/0166-4328(88)90105-2

Hajszan T, Alreja M, Leranth C. 2004. Intrinsic vesicular glutamate transporter 2-immunoreactive input to septohippocampal parvalbumin-containing neurons: novel glutamatergic local circuit cells. Hippocampus 14: 499-509. doi:10.1002/hipo.10195

Hallock HL, Griffin AL. 2013. Dynamic coding of dorsal hippocampal neurons between tasks that differ in structure and memory demand. Hippocampus 23: 169-186. doi:10.1002/hipo.22079

Hallock HL, Arreola AC, Shaw CL, Griffin AL. 2013a. Dissociable roles of the dorsal striatum and dorsal hippocampus in conditional discrimination and spatial alternation T-maze tasks. Neurobiol Learn Mem 100: 108-116. doi:10.1016/j.nlm.2012.12.009

Hallock HL, Wang A, Shaw CL, Griffin AL. 2013b. Transient inactivation of the thalamic nucleus reuniens and rhomboid nucleus produces deficits of a working-memory dependent tactile-visual conditional discrimination task. Behav Neurosci 127: 860-866. doi:10.1037/ a0034653

Herzog CD, Stackman RW, Walsh TJ. 1996. Intraseptal flumazenil enhances, while diazepam binding inhibitor impairs, performance in a working memory task. Neurobiol Learn Mem 66: 341-352. doi:10.1006/nlme .1996 .0074

Kelsey JE, Landry BA. 1988. Medial septal lesions disrupt spatial mapping ability in rats. Behav Neurosci 102: 289-293. doi:10.1037//0735-7044 .102.2.289

Kesner RP, Adelstein TB, Crutcher KA. 1989. Equivalent spatial location memory deficits in rats with medial septum or hippocampal formation lesions and patients with dementia of the Alzheimer's type. Brain Cogn 9: 289-300. doi:10.1016/0278-2626(89)90038-9

Koenig J, Linder AN, Leutgeb JK, Leutgeb S. 2011. The spatial periodicity of grid cells is not sustained during reduced theta oscillations. Science 332: 592-595. doi:10.1126/science.1201685

Leao RN, Targino ZH, Colom LV, Fisahn A. 2015. Interconnection and synchronization of neuronal populations in the mouse medial septum/ diagonal band of Broca. J Neurophysiol 113: 971-980. doi:10.1152/jn .00367 .2014

Maisson DJ, Gemzik ZM, Griffin AL. 2018. Optogenetic suppression of the nucleus reuniens selectively impairs encoding during spatial working memory. Neurobiol Learn Mem 155: 78-85. doi:10.1016/j.nlm.2018.06 .010

Manseau F, Danik M, Williams S. 2005. A functional glutamatergic neurone network in the medial septum and diagonal band area. J Physiol 566: 865-884. doi:10.1113/jphysiol.2005.089664

Mizumori SJ, Perez GM, Alvarado MC, Barnes CA, McNaughton BL. 1990. Reversible inactivation of the medial septum differentially affects two 
forms of learning in rats. Brain Res 528: 12-20. doi:10.1016/0006-8993 (90)90188-h

Morris RG, Garrud P, Rawlins JN, O'Keefe J. 1982. Place navigation impaired in rats with hippocampal lesions. Nature 297: 681-683. doi:10.1038/ 297681a0

Muller C, Remy S. 2018. Septo-hippocampal interaction. Cell Tissue Res 373: 565-575. doi:10.1007/s00441-017-2745-2

Numan R. 1978. Cortical-limbic mechanisms and response control: a theoretical review. Physiol Psychol 6: 445-470. doi:10.3758/BF03326750

Olton DS, Walker JA, Gage FH. 1978. Hippocampal connections and spatial discrimination. Brain Res 139: 295-308. doi:10.1016/0006-8993(78) 90930-7

Olton DS, Walker JA, Wolf WA. 1982. A disconnection analysis of hippocampal function. Brain Res 233: 241-253. doi:10.1016/0006-8993 (82) $91200-8$

Paxinos J, Watson C. 2006. The rat brain in stereotaxic coordinates, 6th ed. Elsevier, New York.

Petersen PC, Buzsaki G. 2020. Cooling of medial septum reveals theta phase lag coordination of hippocampal cell assemblies. Neuron 107: 731-744 e733. doi:10.1016/j.neuron.2020.05.023

Poucet B, Buhot MC. 1994. Effects of medial septal or unilateral hippocampal inactivations on reference and working spatial memory in rats. Hippocampus 4: 315-321. doi:10.1002/hipo.450040315

Rawlins JN, Feldon J, Butt S. 1985. The effects of delaying reward on choice preference in rats with hippocampal or selective septal lesions. Behav Brain Res 15: 191-203. doi:10.1016/0166-4328(85)90174-3

Robinson J, Manseau F, Ducharme G, Amilhon B, Vigneault E, El Mestikawy S, Williams S. 2016. Optogenetic activation of septal glutamatergic neurons drive hippocampal theta rhythms. J Neurosci 36: 3016-3023. doi:10.1523/JNEUROSCI.2141-15.2016

Shaw CL, Watson GDR, Hallock HL, Cline KM, Griffin AL. 2013. The role of the medial prefrontal cortex in the acquisition, retention, and reversal of a tactile visuospatial conditional discrimination task. Behav Brain Res 236: 94-101. doi:10.1016/j.bbr.2012.08.024

Spellman T, Rigotti M, Ahmari SE, Fusi S, Gogos JA, Gordon JA. 2015. Hippocampal-prefrontal input supports spatial encoding in working memory. Nature 522: 309-314. doi:10.1038/nature14445

Stackman RW, Blasberg ME, Langan CJ, Clark AS. 1997. Stability of spatial working memory across the estrous cycle of Long-Evans rats. Neurobiol Learn Mem 67: 167-171. doi:10.1006/nlme.1996.3753
Stewart M, Fox SE. 1990. Do septal neurons pace the hippocampal theta rhythm? Trends Neurosci 13: 163-168. doi:10.1016/0166-2236(90) 90040-h

Stout JJ, Griffin AL. 2020. Representations of on-going behavior and future actions during a spatial working memory task by a high firing-rate population of medial prefrontal cortex neurons. Front Behav Neurosci 14: 151. doi:10.3389/fnbeh.2020.00151

Sun Y, Nguyen AQ, Nguyen JP, Le L, Saur D, Choi J, Callaway EM, Xu X. 2014. Cell-type-specific circuit connectivity of hippocampal CA1 revealed through Cre-dependent rabies tracing. Cell Rep 7: 269-280. doi:10.1016/j.celrep.2014.02.030

Sutherland RJ, Rodriguez AJ. 1989. The role of the fornix/fimbria and some related subcortical structures in place learning and memory. Behav Brain Res 32: 265-277. doi:10.1016/s0166-4328(89)80059-2

Tarnowski BI, Spinale FG, Nicholson JH. 1991. DAPI as a useful stain for nuclear quantitation. Biotech Histochem 66: 297-302.

Toth K, Borhegyi Z, Freund TF. 1993. Postsynaptic targets of GABAergic hippocampal neurons in the medial septum-diagonal band of broca complex. J Neurosci 13: 3712-3724. doi:10.1523/JNEUROSCI .13-09-03712.1993

Wang Y, Romani S, Lustig B, Leonardo A, Pastalkova E. 2015. Theta sequences are essential for internally generated hippocampal firing fields. Nat Neurosci 18: 282-288. doi:10.1038/nn.3904

Whishaw IQ Jarrard LE. 1995. Similarities vs. differences in place learning and circadian activity in rats after fimbria-fornix section or ibotenate removal of hippocampal cells. Hippocampus 5: 595-604. doi:10.1002/ hipo. 450050610

Winson J. 1978. Loss of hippocampal theta rhythm results in spatial memory deficit in the rat. Science 201: 160-163. doi:10.1126/science .663646

Zutshi I, Brandon MP, Fu ML, Donegan ML, Leutgeb JK, Leutgeb S. 2018. Hippocampal neural circuits respond to optogenetic pacing of theta frequencies by generating accelerated oscillation frequencies. Curr Biol 28: $1179-1188$ e1173. doi:10.1016/j.cub.2018.02.061

Received December 4, 2020; accepted in revised form July 30, 2021. 


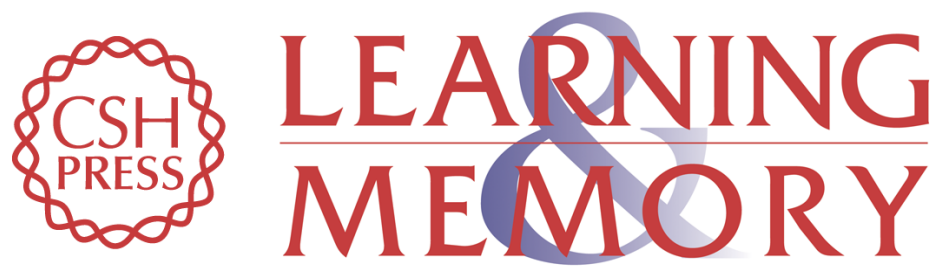

\section{Optogenetic suppression of the medial septum impairs working memory maintenance}

Zachary M. Gemzik, Margaret M. Donahue and Amy L. Griffin

Learn. Mem. 2021, 28:

Access the most recent version at doi:10.1101/Im.053348.120

References This article cites 51 articles, 7 of which can be accessed free at:

http://learnmem.cshlp.org/content/28/10/361.full.html\#ref-list-1

Creative This article is distributed exclusively by Cold Spring Harbor Laboratory Press for the

Commons first 12 months after the full-issue publication date (see

License http://learnmem.cshlp.org/site/misc/terms.xhtml). After 12 months, it is available under a Creative Commons License (Attribution-NonCommercial 4.0 International), as described at http://creativecommons.org/licenses/by-nc/4.0/.

Email Alerting Receive free email alerts when new articles cite this article - sign up in the box at the Service top right corner of the article or click here. 